\title{
Coulomb and Nuclear Potentials Between Deformed Nuclei Applied to the Fusion Process
}

\author{
L. C. Chamon, G. P. A. Nobre, D. Pereira, C. P. Silva, E. S. Rossi Jr., \\ Departamento de Física Nuclear, Instituto de Física da Universidade de São Paulo, \\ Caixa Postal 66318, 05315-970, São Paulo, SP, Brazil \\ L. R. Gasques, \\ Department of Physics \& The Joint Institute for Nuclear Astrophysics, \\ University of Notre Dame, Notre Dame, Indiana, 46556 \\ and B. V. Carlson \\ Departamento de Física, Instituto Tecnologico de Aeronáutica, \\ Centro Técnico Aeroespacial, \\ São José dos Campos, SP, Brazil
}

Received on 22 December, 2004

\begin{abstract}
We obtained theoretical fusion cross sections based on a new and accurate procedure to calculate the Coulomb and nuclear interactions between deformed nuclei. The calculations were performed within the context of the barrier penetration model with zero point motion for the ${ }^{64} \mathrm{Ni}+{ }^{64} \mathrm{Ni}$ system.
\end{abstract}

\section{INTRODUCTION}

It is well known that fusion cross section data for heavy-ion systems show large enhancements at sub-barrier energies in comparison with theoretical predictions from the unidimensional barrier penetration (BP) model [1]. Several attempts to improve the BP model have been performed by considering permanent and/or vibrational deformations of the nuclei (e.g. [2]). Most works in this direction have used approximations for the corresponding deformed potentials. In recent papers [3, 4], we proposed a new procedure to calculate accurately the Coulomb and nuclear interactions between deformed nuclei. We demonstrated that, in certain cases, the usual approximations for the deformed potentials significantly differ from the more accurate results. In the present paper, we compare the results for the fusion cross section when calculating it using the approximate or the more accurate deformed potentials. The calculations were performed for the ${ }^{64} \mathrm{Ni}+$ ${ }^{64} \mathrm{Ni}$ system (data from [5-7]), because this system present very large enhancements in comparison with the predictions of undeformed (unidimensional) BP calculations.

\section{THE COULOMB AND NUCLEAR INTERACTIONS}

Hereafter, we assume the two parameter Fermi $(2 \mathrm{pF})$ distribution to describe the deformed density of a given nucleus $i$

$$
\begin{gathered}
\rho_{i}(\vec{r})=\frac{\rho_{0 i}}{1+\exp \left(\frac{r-R_{i}}{a}\right)}, \\
R_{i}=R_{0 i}+\sum \delta_{\lambda i} Y_{\lambda 0}\left(\Theta_{i}\right),
\end{gathered}
$$

where $\Theta_{i}$ is the angle between $\vec{r}$ and the symmetry axis of the deformation. The corresponding deformation parameter is defined from: $\delta_{\lambda i}=\beta_{\lambda i} R_{0 i}$, and the densities are normalized to

$$
\int \rho(\vec{r}) d \vec{r}=X
$$

where $X$ is the number of protons $Z$ or nucleons $A$ for the charge and matter distributions, respectively. We limit the present study to $\lambda=2$ and 3 . In the vibrational model, the $\beta_{\lambda}$ parameter vary according a Gaussian distribution of probability with standard deviation $\sigma_{\lambda}$. Considering a previous systematics [3], we have adopted the following deformation parameters for the ${ }^{64} \mathrm{Ni}: \sigma_{2}=0.214$ and $\sigma_{3}=0.262$.

The Coulomb interaction between two charge distributions is given by:

$$
V_{C}(R)=\iint \frac{e^{2}}{\left|\vec{R}+\vec{r}_{2}-\vec{r}_{1}\right|} \rho_{1}\left(\vec{r}_{1}\right) \rho_{2}\left(\vec{r}_{2}\right) d \vec{r}_{1} d \vec{r}_{2},
$$

where $\vec{R}$ is the position vector of the center of mass of nucleus 2 measured from that of the nucleus 1 , and describes their relative motion. The direction of the symmetry axis of each deformation relative to $\vec{R}$ is defined by two angles: $\theta_{i}$ and $\phi_{i}$.

The computational resolution of Eq. (4) is very timeconsuming due to the six-dimensional integral involved in the calculation. This becomes a problem in studies where it should be solved several times. Therefore, faster methods to obtain good approximations for the deformed potential are quite convenient. According [3, 4], the Coulomb potential can be obtained with good accuracy from:

$$
V_{C}(R) \approx V_{C}^{(0)}(R)+V_{C o r}(R),
$$

where $V_{C}^{(0)}$ is the non-deformed Coulomb potential, obtained with the corresponding non-deformed densities. The (extensive) expressions for $V_{C o r}(R)$ can be found in Refs. [3, 4]. 
Within the non local model [8-10], the nuclear interaction $V_{N}$ is connected with the folding potential $V_{F}$ through

$$
V_{N}(R, E)=V_{F}(R) e^{-4 \mathrm{~V}^{2} / c^{2}},
$$

where $c$ is the speed of light and $\mathrm{v}$ is the local relative velocity between the two nuclei,

$$
\mathrm{v}^{2}(R, E)=\frac{2}{\mu}\left[E-V_{C}(R)-V_{N}(R, E)\right] .
$$

The folding potential depends on the densities of the nuclei involved in the collision:

$$
V_{F}(R)=\int \rho_{1}\left(\vec{r}_{1}\right) \rho_{2}\left(\vec{r}_{2}\right) v_{N N}\left(\vec{R}-\vec{r}_{1}+\vec{r}_{2}\right) d \vec{r}_{1} d \vec{r}_{2} .
$$

Usually, in Eq. (8) $\rho_{i}$ are the nucleon densities of the nuclei, and $v_{N N}$ is the effective nucleon-nucleon interaction. In many works, the Paris and Reid versions of the M3Y interaction [11] have been assumed for $v_{N N}$. In [10], we demonstrated that the folding type interaction, Eq. (9), produces very similar results in comparison with those from the M3Y:

$$
v_{N N}(r)=\int \rho_{m}\left(r_{1}\right) \rho_{m}\left(r_{2}\right) V_{0} \delta\left(\vec{r}-\overrightarrow{r_{1}}+\overrightarrow{r_{2}}\right) d \overrightarrow{r_{1}} d \overrightarrow{r_{2}},
$$

where $V_{0}=-456 \mathrm{MeV} \mathrm{fm} \mathrm{m}^{3}$ and $\rho_{m}$ is the matter density of the nucleon. Based on the intrinsic charge distribution of the proton in free space, which has been determined by electron scattering experiments, an exponential shape has been assumed for the matter density of the nucleon [10]. Due to the delta function, the folding in Eq. (9) is named as the zerorange approach. We have also defined [10] the matter density of the nucleus $\left(\rho_{M}\right)$ by folding the corresponding nucleon density $\left(\rho_{N}\right)$ with the matter density of the nucleon

$$
\rho_{M}(\vec{r})=\int \rho_{N}\left(\overrightarrow{r^{\prime}}\right) \rho_{m}\left(\vec{r}-\overrightarrow{r^{\prime}}\right) d \overrightarrow{r^{\prime}}
$$

Thus, we distinguish the matter density of the nucleus from the nucleon distribution of the nucleus by taking into account the finite size of the nucleon. By inserting Eqs. (9) and (10) in Eq.(8), the folding potential can be recast in the following form

$$
V_{F}(R)=V_{0} \int \rho_{M 1}\left(\vec{r}_{1}\right) \rho_{M 2}\left(\vec{r}_{1}-\vec{R}\right) d \vec{r}_{1} .
$$

Due to the six-dimensional integral, the numerical resolution of Eq. (8) implies a similar problem as that for the Coulomb potential. Eq. (11) is much faster to calculate because the zero-range approach reduces it to a three-dimensional integral.

In many works (e.g. [2]), the Coulomb and nuclear deformed potentials have been calculated through the following approximate expressions:

$$
\begin{aligned}
V_{C}(R) \approx & Z_{1} Z_{2} e^{2}\left[\frac{1}{R}+\frac{3 \beta_{21} R_{01}^{2}}{5 R^{3}} Y_{20}\left(\theta_{1}\right)+\right. \\
& \frac{3 \beta_{31} R_{01}^{3}}{7 R^{4}} Y_{30}\left(\theta_{1}\right)+\frac{3 \beta_{22} R_{02}^{2}}{5 R^{3}} Y_{20}\left(\theta_{2}\right)- \\
& \left.\frac{3 \beta_{32} R_{02}^{3}}{7 R^{4}} Y_{30}\left(\theta_{2}\right)\right] .
\end{aligned}
$$

$$
\begin{gathered}
V_{F}(R) \approx V_{F}^{(0)}(R) e^{\Delta / a_{P}} \\
\Delta=\beta_{21} R_{01} Y_{20}\left(\theta_{1}\right)+\beta_{31} R_{01} Y_{30}\left(\theta_{1}\right)+ \\
\beta_{22} R_{02} Y_{20}\left(\theta_{2}\right)-\beta_{32} R_{02} Y_{30}\left(\theta_{2}\right),
\end{gathered}
$$

where $V_{F}^{(0)}(R)$ is the folding potential obtained with the undeformed densities and $a_{P}$ is the diffuseness of the potential. Taking into account the approximate exponential shape of the potential in the surface region, the diffuseness involved in Eq. (13) can be estimated by

$$
a_{P}=-V_{F}^{(0)}(R)\left[\frac{d V_{F}^{(0)}}{d R}\right]^{-1} .
$$

However, we have demonstrated that such approximations are not much precise in certain cases [3]. In the next section, we show that the use of these approximations result in fusion cross sections quite different from the values obtained with the more precise deformed potentials.

\section{BARRIER PENETRATION CALCULATIONS}

In the context of the barrier penetration model, the effective potential is a sum of the Coulomb, nuclear and centrifugal parts:

$$
V_{e f f}(R, E)=V_{C}(R)+V_{N}(R, E)+\frac{\ell(\ell+1) \hbar^{2}}{2 \mu R^{2}} .
$$

The BP cross section is associated with the transmitted flux through

$$
\sigma_{B P}(E)=\frac{\pi}{k^{2}} \sum(2 \ell+1) T_{\ell}
$$

For deformed densities, the effective potential and the corresponding BP cross sections depend on the deformation parameters of the nuclei: $\beta_{\lambda i}, \theta_{i}$, and $\phi_{i}$. In this work, we assume the sudden approximation where such parameters remain constant during the collision. However, from one collision to another, these parameters can vary according their distributions of probability:

$$
\begin{gathered}
P(\theta)=\frac{1}{2} \sin (\theta), \\
P(\phi)=\frac{1}{2 \pi}, \\
P(\beta)=\frac{1}{\sqrt{2 \pi} \sigma} e^{-\frac{\beta^{2}}{2 \sigma^{2}}} .
\end{gathered}
$$

We have calculated BP cross sections by obtaining the corresponding average over the possible configurations of the deformation parameters. We have used a Monte Carlo method, where the values of $\beta_{\lambda i}, \theta_{i}$, and $\phi_{i}$ are obtained randomly according their distributions of probability. Fig. 1 presents the 
fusion data for the ${ }^{64} \mathrm{Ni}+{ }^{64} \mathrm{Ni}$ system. There are different data sets with cross sections that differ by a factor of about three in the sub-barrier energy region. These different data sets have been represented by different symbols. The dotted line in Fig. 1 represents the cross sections obtained with the undeformed potential, while the dashed and solid lines represent the results obtained with the approximate and with the more accurate expressions for the deformed potentials, respectively. Clearly, the deformation largely enhances the cross section in the sub-barrier region and almost does not affect it in the region above the barrier. The use of the approximations results in fusion cross sections quite different from the values obtained with the more precise deformed potentials.

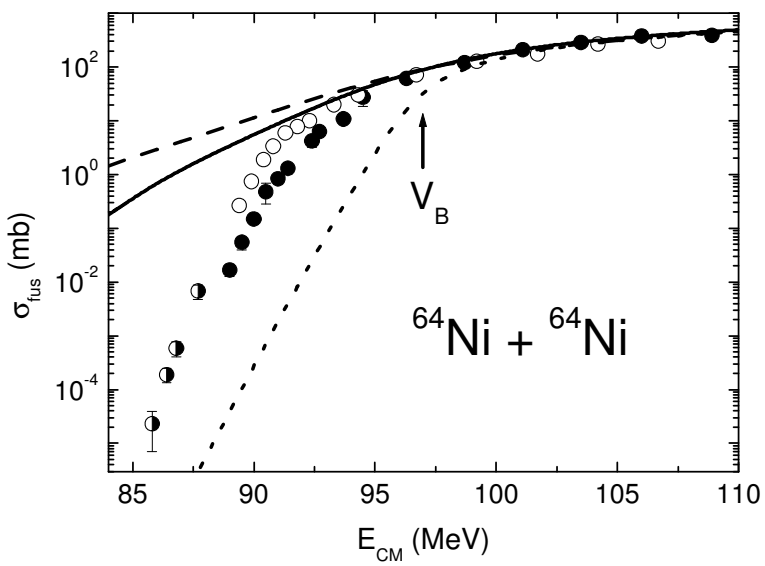

FIG. 1: Fusion cross sections for the ${ }^{64} \mathrm{Ni}+{ }^{64} \mathrm{Ni}$ system. The dotted lines represent BP calculations considering non-deformed densities. The other lines correspond to deformed densities obtained with the usual approximate expressions for the potentials (dashed line) and with the more precise expressions assumed in this work (solid line). The arrow represents the position of the s-wave barrier height $\left(V_{B}\right)$ obtained with the non-deformed interaction.
Our calculations with the accurate potentials show that the vibrational model is in a reasonable agreement with the fusion data in a level so down as about $1 \mathrm{mb}$. Below this level, for extreme sub-barrier energies, the data present a decrease much steeper than the theoretical predictions. Similar hindrance of the data compared to predictions of coupled-channel calculations has already been observed for several systems [7, 12, 13].

\section{SUMMARY AND CONCLUSION}

We have used an accurate method for calculating deformed potentials to study the fusion process for the ${ }^{64} \mathrm{Ni}+{ }^{64} \mathrm{Ni}$ systems within the context of the barrier penetration model with zero point motion. We demonstrated that the accurate method provides fusion cross sections that significantly differ from those obtained with the usual approximations for the deformed potentials. The accurate method gives cross section predictions that reproduce the order of magnitude of the data to a level about $1 \mathrm{mb}$. The hindrance of the fusion data below this level, observed in extreme sub-barrier energies, is still an open problem. This phenomenon is quite important for reactions of astrophysical interest, as these usually take place in such very low energy region.

\section{Acknowledgments}

This work was partially supported by Financiadora de Estudos e Projetos (FINEP), Fundação de Amparo à Pesquisa do Estado de São Paulo (FAPESP), Conselho Nacional de Desenvolvimento Científico e Tecnológico $(\mathrm{CNPq})$, and The Joint Institute for Nuclear Astrophysics (JINA) NSF PHY 0216783.
[1] L. C. Vaz and J. M. Alexander, Phys. Rev. C 18, 2152 (1978)

[2] H. Esbensen, Nucl. Phys. A 352, 147 (1981).

[3] L. C. Chamon, G. P. A. Nobre, D. Pereira, E. S. Rossi Jr., C. P. Silva, L. R. Gasques, and B. V. Carlson, Phys. Rev. C 70, 014604 (2004).

[4] B. V. Carlson, L. C. Chamon, and L. R. Gasques, to appear in Phys. Rev. C.

[5] M. Beckerman, M. Salomaa, A. Sperduto, J. D. Molitoris, and A. DiRienzo, Phys. Rev. C 25, 837 (1982).

[6] M. Stefanini, K. M. Varier, H. Zhang, F. Scarlassara, S. Beghini, G. Montagnoli, L. Miller, G. F. Segato, F. Soramel, and C. Signorini, Nucl. Phys. A 609, 91 (1996).

[7] C. L. Jiang, K. E. Rehm, R. V. F. Janssens, H. Esbensen, I. Ahmad, B. B. Back, P. Collon, C. N. Davids, J. P. Greene, D. J. Enderson, G. Mukherjee, R. C. Pardo, M. Paul, T. O. Pennington, D. Seweryniak, S. Sinha, and Z. Zhou, Phys. Rev. Lett. 93 012701 (2004).
[8] M. A. Cândido Ribeiro, L. C. Chamon, D. Pereira, M. S. Hussein and D. Galetti, Phys. Rev. Lett. 78, 3270 (1997).

[9] L. C. Chamon, D. Pereira, M. S. Hussein, M. A. Cândido Ribeiro and D. Galetti, Phys. Rev. Lett. 79, 5218 (1997).

[10] L. C. Chamon, B. V. Carlson, L. R. Gasques, D. Pereira, C. De Conti, M. A. G. Alvarez, M. S. Hussein, M. A. Candido Ribeiro, E. S. Rossi Jr., and C. P. Silva, Phys. Rev. C 66, 014610 (2002).

[11] G. R. Satchler and W. G. Love, Phys. Rep. 55, 183 (1979).

[12] C. L. Jiang, H. Esbensen, K. E. Rehm, B. B. Back, R. V. F. Janssens, J. A. Caggiano, P. Collon, J. Greene, A. M. Heinz, D. J. Henderson, I. Nishinaka, T. O. Pennington, and . Seweryniak, Phys. Rev. Lett. 89, 052701 (2002).

[13] C. L. Jiang, H. Esbensen, B. B. Back, R. V. F. Janssens, and K. E. Rehm, Phys. Rev. C 69, 014604 (2004). 